\title{
ARTICLE
}

\section{Impact of ADCYAP1R1 genotype on longitudinal fear conditioning in children: interaction with trauma and sex}

\author{
Tanja Jovanovic (iD) ${ }^{1}$, Anaïs F. Stenson (iD) ${ }^{1}$, Nadia Thompson ${ }^{2}$, Aimee Clifford ${ }^{2}$, Alisha Compton ${ }^{2}$, Sean Minton ${ }^{2}$, Sanne J. F. van Rooij (iD) \\ Jennifer S. Stevens (D) $^{2}$, Adriana Lori ${ }^{2}$, Nicole Nugent ${ }^{3}$, Charles F. Gillespie iD $^{2}$, Bekh Bradley ${ }^{2,4}$ and Kerry J. Ressler ${ }^{5}$
}

Dysregulated fear conditioned responses have been associated with PTSD in adults, with increased fear-potentiated startle (FPS) serving as a potential intermediate phenotype for PTSD risk. This phenotype has also been associated with stress-related ADCYAP1R1 gene variants in adult women. However, FPS and genotype have not yet been examined during development. The aim of this study was to examine developmental changes in fear conditioning, and to see whether these changes were impacted by genotype and trauma. Differential fear conditioning using FPS was tested in $n=63$ children ages 8-13 at two visits (V1, V2) 1 year apart. Startle response was measured using electromyograph recordings of the eyeblink muscle. The rs2267735 SNP of the ADCYAP1R1 gene was extracted from genome-wide (GWAS) analyses. Trauma exposure was assessed using the Violence Exposure Scale-Revised (VEX-R). We found significant Visit by Genotype interactions, with CC genotype increasing FPS from V1 to V2. At V2 there was a Genotype by Violence interaction, with higher FPS in the CC vs G allele groups among those with higher violence exposure $(F=17.46, p=0.0002)$. Females with the CC genotype had higher FPS compared to $\mathrm{G}$ allele females $(F=12.09, p=0.002)$; there were no effects of genotype in males. This study showed Gene $\times$ Environment $\times$ Development and Gene $\times$ Sex effects of $A D C Y A P 1 R 1$ in a high-risk pediatric population. Those with the CC genotype and high levels of violence exposure, as well as females with the CC genotype, showed the greatest conditioned fear responses in adolescence.

Neuropsychopharmacology (2020) 45:1603-1608; https://doi.org/10.1038/s41386-020-0748-2

\section{INTRODUCTION}

Fear or threat conditioning is a form of Pavlovian conditioning in which a neutral conditioned stimulus (CS) is repeatedly paired with an aversive unconditioned stimulus (US). Differential fear conditioning involves two stimuli: the reinforced CS becomes a threat signal $(\mathrm{CS}+)$ and elicits a fear response, while a second, non-reinforced CS becomes the safety signal (CS-). Dysregulated fear conditioned responses, measured using psychophysiological indices, such as skin conductance response (SCR) and fearpotentiated startle (FPS), have been associated with trauma- and anxiety-related disorders in adults who have higher fear responses to threat signals $[1-3]$, as well as safety signals $[4,5]$ compared to controls.

While age-related changes have been observed in fear conditioning with respect to both threat and safety signals, these have been almost exclusively cross-sectional studies [6-9]. Results indicate that discrimination between $\mathrm{CS}+$ and $\mathrm{CS}-$ develops around age $10[6,7]$. Some studies have compared children to adults and found that children show higher fear responses to threat and more generalization of fear $[10,11]$, also indicating that inhibition of fear may occur later in development. Studies that have compared adolescents to adults report similar patterns, in that adolescents show less $\mathrm{CS}+/ \mathrm{CS}$ - discrimination relative to adults $[12,13]$.
One longitudinal study examined fear conditioning with SCR annually from ages 3 to 8 , and found that discrimination between CS + and CS- emerged at age 8 [14]. We are not aware of longitudinal studies of fear conditioning during the transition from middle childhood to adolescence ( 8-13 years old), when the prevalence of psychopathology increases and sex differences in risk emerge [15]. The goal of our study was to examine whether there were developmental changes in fear conditioning in 8- to 13-year-old children at elevated risk for trauma exposure [16], and if those changes were influenced by genes and adverse environment.

Data from adults suggest that FPS responses may serve as an intermediate phenotype for trauma-related psychopathology, such as post-traumatic stress disorder (PTSD) [17]. Such phenotypes may be particularly useful in examining potential neurobiological mechanisms for genetic risk factors, especially during development [18]. While several candidate genes have been associated with PTSD, these have not been replicated in large genome-wide association studies (GWAS) [19, 20]. In fact, candidate gene studies are largely underpowered to detect complex clinical phenotypes, such as PTSD. However, focusing on intermediate phenotypes, such as fear responses, may reduce some of the complexity and allow for improved power despite smaller sample sizes [5].

\footnotetext{
${ }^{1}$ Department of Psychiatry and Behavioral Neurosciences, Wayne State University, Detroit, MI, USA; ${ }^{2}$ Department of Psychiatry and Behavioral Sciences, Emory University, Atlanta, GA, USA; ${ }^{3}$ Departments of Psychiatry and Pediatrics Brown Medical School, Providence, RI, USA; ${ }^{4}$ Atlanta VA Medical Center, Decatur, GA 30033 , USA and ${ }^{5}$ McLean Hospital, Harvard Medical School, Belmont, MA 02478, USA

Correspondence: Tanja Jovanovic (tjovanovic@med.wayne.edu)
}

Received: 2 January 2020 Revised: 17 June 2020 Accepted: 22 June 2020 Published online: 26 June 2020 
A single nucleotide polymorphism (SNP) in the gene coding for pituitary adenylate cyclase-activating polypeptide (PACAP) receptor (ADCYAP1R1), rs2267735, has been repeatedly associated with PTSD [21-25] and a number of neurobiological intermediate phenotypes [25, 26], including FPS in adult women [27]. Neuroimaging studies have found that the CC genotype of rs2267735 is associated with greater amygdala activity to fear stimuli in women [26]. Convergent preclinical data in rats shows that PACAP modulates fear conditioning and that ADCYAP1R1 is highly expressed in the prefrontal cortex in females [28]. A recent meta-analysis of human studies that analyzed this SNP concluded that there is evidence for sex differences in an association of ADCYAP1R1 with PTSD, with a robust relationship between $A D C Y A P 1 R 1$ and PTSD observed in female samples [29]. Taken together, these studies suggest that the ADCYAP1R1 genotype may impact fear-relevant intermediate phenotypes of risk for PTSD. While we have shown in a previous study that the CC genotype is associated with increased dark-enhanced startle in children [30], this SNP has not been examined in children using FPS as an outcome. Moreover, the current study is the only one to longitudinally investigate the association between fear conditioning, risk genotype, and sex differences in children.

Childhood maltreatment has been found to moderate ADCYAP1R1 rs2267735 risk for PTSD in women [23], indicating a $\mathrm{G} \times \mathrm{E} \times$ Development effect. The only study that has examined $A D C Y A P 1 R 1$ rs 2267735 and violence exposure in children (ages 6-14) was focused on asthma as an outcome [31], and found that exposure to violence increased methylation of this gene. To date there have been no studies that we know of that have reported on the $\mathrm{G} \times \mathrm{E}$ interaction of $A D C Y A P 1 R 1$ and early trauma in children and adolescents.

The goals of the current study were to address this knowledge gap by investigating $\mathrm{G} \times \mathrm{E}$ effects on fear conditioning in 8- to 13year-old children at high risk for trauma exposure longitudinally. We hypothesized that: (1) discrimination between CS + and CSwould improve over time from baseline to follow-up, (2) CC genotype of $A D C Y A P 1 R 1$ would be associated with higher levels of FPS, and (3) trauma exposure would interact with genotype to increase FPS in the CC genotype group. Finally, (4) we hypothesized that sex would interact with genotype, such that in females the CC genotype would be associated with higher FPS.

\section{METHOD}

Longitudinal assessments

The current study was part of an ongoing longitudinal study of trauma exposure in children as part of the Grady Trauma Project in Atlanta, GA. Visit 1 (V1) included consent, saliva sampling for DNA, fear conditioning, and self-report and maternal assessment on questionnaires. Visit 2 (V2) occurred $\sim$ year later $(M=$ 11.96 months) and fear conditioning and assessments were repeated.

\section{Participants}

Study participants were 63 African-American children (29 females) who participated at both visits. Mothers were recruited from an ongoing study of PTSD and trauma exposure. In order to be eligible for the study, child participants had to be between 8 and 13 years of age at V1 and willing to participate; exclusion criteria included diagnosis of autism spectrum disorder, bipolar disorder, psychotic disorder, or cognitive disability. A subset of V1 startle data from this study have been published previously [6, 8, 32]; however, the current study also included individuals that had (1) complete startle data at both visits, (2) ADCYAP1R1 rs2267735 SNP data from the GWAS analysis, and (3) available information about violence exposure. Of the 120 participants who completed startle on $\mathrm{V} 1,104$ returned for $\mathrm{V} 2$ ( $86.7 \%$ retention). Of those, 16 had incomplete V2 startle, and another 25 had data loss due to mostly computer error or noisy data. The final sample size was 63. Prior to their participation, all mothers signed informed consent as well as parental permission for their children, and the children provided study assent approved by the Institutional Review Board and the Research Oversight Committee.

Fear conditioning

Participants were seated in a sound attenuated booth and asked to remain still and look at a computer monitor $\sim 1 \mathrm{~m}$ in front of them. The acoustic startle probe was a 106-dB [A] SPL, 40-ms burst of broadband noise delivered binaurally through headphones. The US was an aversive airblast directed to the larynx at an intensity of 80 p.s.i. and $100 \mathrm{~ms}$ in duration. This US has been used successfully in our lab to elicit fear conditioned responses in pediatric populations [8]. The CSs were colored shapes presented on a computer monitor using Superlab 4.5 presentation software (Cedrus, Inc.) for $6 \mathrm{~s}$ prior to the delivery of the startle probe, and co-terminated with the US $500 \mathrm{~ms}$ after the presentation of the startle stimulus. The V2 session included a novel set of colored shapes for the CSs that were not previously seen by the participants. In both sessions (V1 and V2) the CS + was paired with the airblast $100 \%$ of the time, and the CS - was never paired with the airblast. The session consisted of a habituation block in which three CS trials of each type were presented without the US and three blocks of acquisition, each with three reinforced CS + trials, 3 CS - trials, and three noise alone (NA) trials, for a total of 36 trials. In all phases of the experiment, inter-trial intervals will be randomized between 9 and $22 \mathrm{~s}$; the acquisition session lasted $13 \mathrm{~min}$.

\section{Startle measurement}

Electromyographic (EMG) data from the eyeblink muscle contraction in response to the startle probe were sampled at $1000 \mathrm{~Hz}$ and amplified using the Bionomadix wireless EMG module of the Biopac MP150 for Windows (Biopac Systems, Inc., Aero Camino, CA). EMG activity was recorded from two $5 \mathrm{~mm} \mathrm{Ag} / \mathrm{AgCl}$ electrodes placed over the orbicularis oculi muscle, $\sim 1 \mathrm{~cm}$ under the pupil and $1 \mathrm{~cm}$ below the lateral canthus. A ground electrode was placed behind the ear. The impedances for all participants were less than $6 \mathrm{k} \Omega$. The acquired data were rectified and visually inspected for artifacts in MindWare software (MindWare Technologies, Inc) and exported for statistical analyses. The EMG signal was filtered in MindWare with low- and high- frequency cutoffs at 28 and $500 \mathrm{~Hz}$, respectively. Startle magnitude was assessed as the peak amplitude of the EMG contraction 20 to $200 \mathrm{~ms}$ following the sound probe.

\section{Genotyping}

DNA was extracted from saliva in Oragene collection vials (DNA Genotek Inc, Ontario, Canada) using standard procedure by AKESOgen (Akesogen.com). Genome-wide SNP genotyping was conducted using the Illumina Infinium PsychArray Beadchip. Samples were processed at Akesogen, spread in three runs and re-clustered together before calls. Standard quality control of the genome-wide data was performed using PLINK (version 1.9) [33] removing individuals with greater than $5 \%$ missing data and removing one in each pair of related individuals with an identity by descent proportion $>0.12$ (indicating cousins or a closer relation). We removed SNPs with call rates $<95 \%$, minor allele frequency $<0.05$, and deviation from Hardy-Weinberg proportions. Principalcomponent analysis (PCA) was then performed to infer African-American ancestry and remove outlier subjects. Based on PCA, we did not observe any batch effects from the different runs. PC eigenvectors of the genetic relationship matrix were calculated by using $\sim 50,000$ independent SNPs. The rs2267735 SNP was extracted from the non-imputed genome-wide data (HWE $p>0.4$ )

\section{Assessments}

Trauma exposure in children was assessed using the Violence Exposure-Revised (VEX-R) [34]. The VEX-R is a 22-item cartoon 
self-report measure of children's exposure to violence, which has been validated in the Grady Trauma Project and is highly correlated with self-reported PTSD symptoms in children [16]. VEX-R at V2 was used in the analyses to capture cumulative trauma exposure. PTSD symptoms were assessed using the UCLA Reaction Index [35]. Although the trauma exposure was moderately high (Supplemental Fig. 1), with a median of six traumatic events, the PTSD symptoms in the sample were low, and did not increase between visits $(\mathrm{V} 1=16.01, \mathrm{~V} 2=17.94, p=0.128)$. We measured pubertal status at each visit with the Pubertal Development Scale (PDS) [36]. The five-item scale has female and male versions. Median internal consistency was good, $a=0.77$ (range: $0.68-0.83$ ) and has been associated with fear conditioning in girls [37].

Statistical analyses

We first tested that the participants were demonstrating potentiated acoustic startle responses to the CS+ by comparing startle amplitude on NA, CS+, and $\mathrm{CS}-$ trials using a Block (4 levels: Hab, Acq1, Acq2, Acq3) by Trial Type (3 levels: NA, CS+, CS-) repeated-measures analysis of variance (RM-ANOVA). Visit (2 levels: V1, V2) was also included in the RM-ANOVA as a withinsubjects factor. FPS was indexed by calculating a difference score for each CS type, in order to account for individual differences in startle magnitude as well as startle habituation. This value was derived by subtracting startle magnitude during NA trials from CS trials. Hypothesis 1, that discrimination between $\mathrm{CS}+$ and $\mathrm{CS}-$ would improve from V1 to V2, was tested using the above Visit $X$ Block $\times$ Trial Type RM-ANOVA with startle amplitude as the dependent variable. Hypothesis 2, that risk genotype for ADCYAP1R1 would be associated with higher levels of FPS, was tested using a mixed model ANOVA of Visit (2 levels: V1, V2) $\times C S$ Type (2 levels: $\mathrm{CS}+, \mathrm{CS}-$ ) $\times$ Genotype ( 2 levels: CC, CG/GG) with FPS during late acquisition as the dependent variable. Late acquisition averaged FPS across blocks 2 and 3 in order to better capture the learning effects across blocks. For genotype analyses, the heterozygous genotypes were combined with the GG group as in previous studies of $\operatorname{rs} 2267735[23,27,30]$, testing for a dominant model of penetrance. The top two principle components ( $P C S$ ) derived from the GWAS analysis accounting for ancestry markers were used as covariates in the analysis. Age and sex were also entered in the analysis as covariates to assess whether effects of visit were independent of increased age, and whether the effects of genotype were independent of sex.

Hypothesis 3, that trauma exposure would interact with genotype to increase FPS in the CC genotype group, was tested using a median split of the VEX-R total score to derive Higher and Lower Violence Exposure groups that were entered into the ANOVA as a between-groups factor. The top two PCs, sex, and age were added as covariates. To test Hypothesis 4, that CC genotype would be associated with higher FPS specifically in females, we repeated the Visit $\times$ CS Type $\times$ Genotype mixed model ANOVA with adding sex as a between-groups factor. In addition to PCs and age, total PDS score was also included as a covariate to account for sex differences in stage of pubertal development. All higher-level interactions were followed up by univariate ANOVAs. All analyses were performed using SPSS 25.0 for Windows, and alpha was set to 0.05. Partial eta squared $\left(\eta^{2}\right)$ is presented for estimates of effect size.

\section{RESULTS}

Longitudinal effects on fear conditioning

A RM-ANOVA of Visit $\times$ Block $\times$ Trial Type resulted in a significant main effect of Visit $\left(F=4.41, p=0.04, \eta^{2}=0.07\right)$, and a Block by Trial Type interaction for NA vs CS $+\left(F=7.80, p=0.007, \eta^{2}=0.12\right)$ and $C S+$ vs $C S-\left(F=6.55, p=0.01, \eta^{2}=0.11\right)$, indicating that fear potentiation to CS+ as well as discrimination between CS+ and CS- developed in blocks 2 and 3 of conditioning (see Fig. 1). Averaging startle amplitude across these last two blocks showed a significant potentiation of startle to CS + compared to NA ( $F=$ $\left.8.29, p=0.006, \eta^{2}=0.13\right)$ and significant discrimination between $\mathrm{CS}+$ and $\mathrm{CS}-\left(F=4.08, p=0.048, \eta^{2}=0.07\right)$. Importantly, the effect of Visit was not related to retention of the original fear conditioning, as there was no potentiation to CS+ or discrimination during the habituation phase at V2. While there was an overall effect of Block by Trial Type, there was no Visit by Block by Trial Type interaction, indicating that learning occurred in the same pattern across both visits. The absence of a Visit by Trial Type interaction effect suggests that there were no facilitation effects on fear conditioning after 1 year.

\section{ADCYAP1R1 genotype effects on fear conditioning}

The demographic data for each Genotype group are listed in Table 1. To test Hypothesis 2, we examined FPS during late acquisition (average FPS of blocks 2 and 3 of acquisition) in a mixed RM-ANCOVA with Visit $\times$ CS Type as within-subjects factors, and Genotype as the between-groups variable. The top two GWAS PCs, sex, and age were used as covariates in the analyses. We found a significant Visit by Genotype interaction $\left(F=5.23, p=0.03, \eta^{2}=\right.$ $0.11)$, with CC associated with higher FPS compared to $G$ carriers at V2 $\left(F=6.47, p=0.014, \eta^{2}=0.11\right)$ but not at the baseline visit $(p=$ 0.68 ), see Fig. 2. There was no interaction between Genotype and CS Type, suggesting that the CC genotype was associated with higher startle to both threat and safety signals.

Impact of trauma and genotype

To examine the impact of trauma exposure we analyzed the interaction of genotype and trauma on late acquisition FPS. We conducted a mixed RM-ANCOVA with Visit and CS Type as

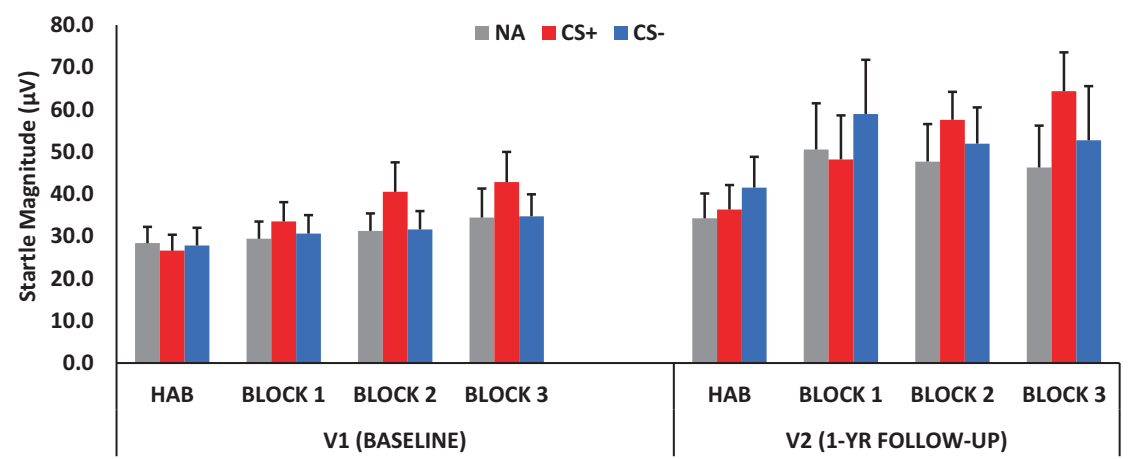

Fig. 1 Mean + SE startle magnitude during differential fear conditioning at baseline and follow-up. There was a significant main effect of Visit, and a Block by Trial Type interaction for NA vs CS + , and CS + vs CS-, indicating that fear potentiation to CS + as well as discrimination between CS + and CS- developed in blocks 2 and 3 of conditioning. NA noise alone, CS + reinforced conditioned stimulus, CS- nonreinforced conditioned stimulus, HAB habituation. 
Table 1. Demographic characteristics of the sample.

\begin{tabular}{|c|c|c|}
\hline & $\begin{array}{l}\text { ADCYAP1R1 CC } \\
\text { genotype } \\
N=24\end{array}$ & $\begin{array}{l}\text { ADCYAP1R1 GG/GC } \\
\text { genotype } \\
N=39\end{array}$ \\
\hline \multicolumn{3}{|l|}{ Age (M, SD) } \\
\hline V1 (baseline) & $10.46(1.41)$ & $10.09(1.55)$ \\
\hline V2 (1-year follow-up) & $11.56(1.72)$ & $10.99(1.64)$ \\
\hline \multicolumn{3}{|l|}{$\operatorname{Sex}(N, \%)$} \\
\hline Female & $10(41.7 \%)$ & 19 (48.7\%) \\
\hline Male & $14(58.3 \%)$ & 20 (51.3\%) \\
\hline \multicolumn{3}{|l|}{ Income $(N, \%)$} \\
\hline Below $\$ 1,000 /$ month & $12(50 \%)$ & $18(47.4 \%)$ \\
\hline Above $\$ 2,000 /$ month & $6(25 \%)$ & 5 (13.2\%) \\
\hline \multicolumn{3}{|l|}{ Violence exposure (V2) } \\
\hline High violence & $9(45.0 \%)$ & 27 (73\%) \\
\hline Low violence & $11(55.0 \%)$ & $10(27 \%)$ \\
\hline \multicolumn{3}{|l|}{ PTSD criteria $(N, \%)$} \\
\hline V1 (baseline) & $6(28.6 \%)$ & $6(15.4 \%)$ \\
\hline V2 (1-year follow-up) & $5(26.3 \%)$ & $10(25.6 \%)$ \\
\hline
\end{tabular}

within-subjects factors, and Genotype and Violence Level as the between-groups variables, while controlling for PCs, sex, and age. We found a significant three-way interaction of Genotype $\times$ Violence Level $\times$ Visit $\left(F=4.17, p=0.049, \eta^{2}=0.11\right)$, and a twoway interaction of Genotype and Visit $\left(F=7.63, p=0.009, \eta^{2}=\right.$ $0.18)$. We followed up the 3-way interaction within each visit and found a significant Genotype by Violence Level interaction $(F=$ $\left.8.82, p=0.005, \eta^{2}=0.16\right)$ at V2 but not V1 $(p=0.50)$, see Fig. 3. Stratifying by Lower vs Higher Violence levels showed that FPS at V2 was increased in the CC group only among those with higher violence exposure $\left(F=17.46, p=0.0002, \eta^{2}=0.38\right)$. We then added PTSD symptoms at V2 as a covariate, and the Genotype effect remained significant $(p=0.002)$.

Sex differences and genotype

Given our hypotheses about sex differences, we repeated the above analyses of late acquisition FPS, using a mixed RM-ANCOVA with Visit and CS Type as within-subject, and Genotype, and Sex as the between-groups variables. In these analyses, we also covaried for PCs, age, and pubertal development, since girls had higher PDS scores $(M=2.11, \mathrm{SD}=0.57)$ than boys $(M=1.74, \mathrm{SD}=0.43 ; F=$ $8.12, p=0.006)$. Again, there was a significant interaction of Visit and Genotype $\left(F=4.94, p=0.032, \eta^{2}=0.11\right)$, so we repeated the analysis separately for $\mathrm{V} 1$ and $\mathrm{V} 2$. There were no significant effects of Genotype or Sex at V1. However, at V2 we again found a significant two-way interaction of Genotype by $\operatorname{Sex}(F=4.52, p=$ $0.038, \eta^{2}=0.08$ ), see Fig. 4 , in addition to a main effect of Genotype $\left(F=6.25, p=0.016, \eta^{2}=0.11\right)$. Stratifying the analyses by Sex showed that females with the CC genotype had higher FPS compared to females who were $\mathrm{G}$ carriers $\left(F=12.09, p=0.002, \eta^{2}\right.$ $=0.35$ ), while there were no effects of genotype in males.

\section{DISCUSSION}

Alterations in fear conditioning have been strongly linked with trauma-related disorders [38, 39], and the ADCYAP1R1 gene has been associated with fear conditioning in human and animal studies $[27,28]$, however, no study we are aware of has investigated a $G \times E$ interaction on fear conditioning during development. This is the first longitudinal study that examines genotype and trauma exposure in relation to fear conditioning during the transition from middle childhood to adolescence, when both biological and environmental factors contribute to elevated risk for psychopathology [40]. Our results showed fear discrimination between threat and safety signals; this effect did not interact with visit, suggesting that fear

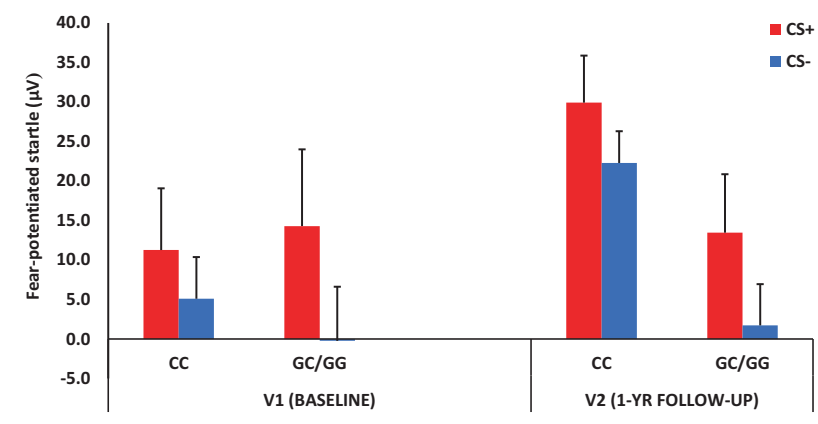

Fig. 2 Mean + SE fear-potentiated startle during late acquisition (blocks 2 and 3 of conditioning). Fear-potentiated startle was calculated as a difference score between startle magnitude to the CS and startle magnitude to NA. We found a significant Visit by Genotype interaction, with CC associated with higher FPS compared to G carriers at V2 but not at the baseline visit. CS+ reinforced conditioned stimulus, CS- non-reinforced conditioned stimulus.

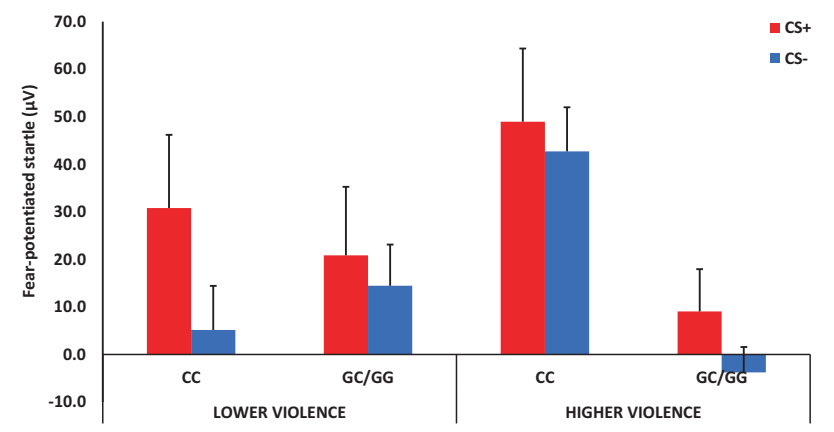

Fig. 3 Mean + SE startle magnitude during differential fear conditioning at visit 2. There was a significant interaction effect of Violence by Genotype, with CC genotype associated with higher FPS compared to G carriers at in the Higher Violence exposure group. CS+ reinforced conditioned stimulus, CS- non-reinforced conditioned stimulus.

learning was already evident at the baseline visit. Children's rs2267735 genotype did interact with visit: the CC genotype group showed increased FPS at V2 compared to the G allele group. Further, high levels of violence exposure and female sex interacted with genotype and were associated with higher FPS in the CC group only at V2. These findings suggest that sex, genotype, and adverse environmental exposures may jointly impact fear learning during early adolescence.

The lack of longitudinal changes in fear discrimination in the overall sample was somewhat surprising. However, although the children were on average a year older at the follow-up visit, V1 included adolescents who show robust fear conditioning and discrimination between danger and safety $[6,8]$. Notably, children did not appear to retain knowledge of the fear conditioning from one visit to the next. There were no interactions between visit and trial type or block and trial type, indicating that there were no carryover effects in either the start of the conditioning session or facilitation of discrimination at V2. At both visits, fear potentiation to the CS + and discrimination between CS+ and CS - emerged during the last two blocks of conditioning (Fig. 1). Interestingly, there was an overall increase in startle amplitude between visits, which may reflect context conditioning to the testing booth, although the lack of increase between V1 and V2 during the habituation phase suggests that this might not have been the case.

The interaction of visit with genotype suggests that increases in fear responses emerge during development in children who may be more genetically susceptible. The effect of genotype was significant only at the second visit. This genotype-by-visit 


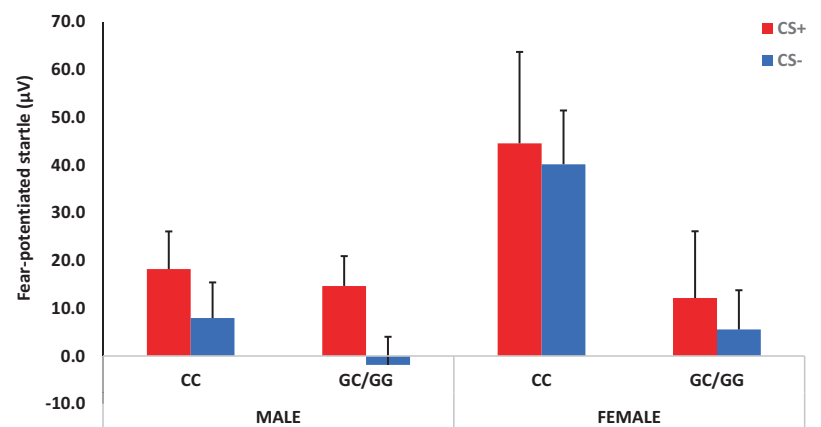

Fig. 4 Mean + SE startle magnitude during differential fear conditioning at visit 2. There was a significant interaction effect of Sex by Genotype, with CC genotype associated with higher FPS compared to $\mathrm{G}$ carriers in females. CS+ reinforced conditioned stimulus, CS- non-reinforced conditioned stimulus.

interaction remained significant after co-varying for age, suggesting that other aspects of developmental change, such as pubertal development or new environmental exposures, may interact with genotype to confer risk. Importantly, this suggests that crosssectional studies would not necessarily observe the same effects. Previous research has linked the CC genotype of rs2267735 with PTSD in adult women [29], yet it has not been clear whether risk for PTSD is related to developmental processes, as would be likely if the association was related to sex hormones.

Neuroimaging studies have implicated the CC genotype in activation of fear circuitry [26]; this same circuitry shows increased activation with age in pediatric PTSD [41]. The amygdala and prefrontal cortex demonstrate PTSD-related developmental changes [42], as well as changes in connectivity between childhood and adolescence [43] and underlie fear conditioning [5]. In our study, however, PTSD symptoms did not account for the association between CC genotype and FPS, suggesting that increased fear response may be an intermediate phenotype of early brain-based risk even before PTSD symptoms emerge.

Fear conditioned responses have been associated with the $A D C Y A P 1 R 1$ gene only in adults. Our previous study that examined children [30] found that the CC genotype associated with increased dark-enhanced startle, but this paradigm does not involve fear learning and most of the participants were prepubertal. In the current study, we found a sex difference in that girls with the CC genotype showed increased FPS compared to girls who had the $G$ allele, whereas there was no effect of genotype in boys. It should be noted that the genotype by sex interaction was only found at the later time point in development; given the lack of a three-way interaction of visit $\times$ genotype $\times$ sex, the analyses within the second visit should be considered exploratory. Nevertheless, it is likely that the sex differences may begin to emerge early and are only evident later on though pubertal development. This finding supports the literature on $A D C Y A P 1 R 1$ interaction with estrogen, as the gene contains an estrogen response element [44]. While it is possible that pubertal status impacted the sex difference findings, as girls had higher scores on pubertal development than boys, the effect of sex remained significant even after controlling for PDS.

We also found an interaction of genotype with trauma exposure, in that the CC genotype was associated with higher FPS in those with higher levels of exposure to violence. This finding also replicates previous $G \times E$ studies that have found that the ADCYAP1R1 CC genotype increased PTSD symptoms in adult women with higher trauma exposure $[22,23]$. While one study found that high childhood maltreatment was associated with greater PTSD prevalence in the CC genotype group, these results were based on retrospective data collected in adults [23]. A previous study found that childhood maltreatment was associated with blunted fear conditioned responses [45], however, a prospective study of adolescents found that early childhood adversity had a smaller effect on startle to safety than adolescent trauma exposure [46]. In the present study we found an effect of violence exposure reported at the same time point as the FPS testing (V2), suggesting that adolescents may be most impacted by trauma that is relatively recent. It is important to note that the median number of trauma types reported in our sample was 6 , therefore even the lower violence exposure group had non-trivial exposure.

There are several limitations to the current study. First, the sample size is small, especially when genotype and trauma exposure, and genotype and sex, are taken into account. However, the unique longitudinal characteristics and deep phenotyping of this cohort make our results an important preliminary finding. For this reason, we included effect sizes in order to better understand the impact of genotype and to support future replication studies. Second, the longitudinal follow-up timeframe, i.e. 1 year later, may be too short to see clinical changes. In fact, the majority of the participants in the study did not meet diagnostic criteria threshold for PTSD despite high rates of trauma exposure-longer-term follow-up data may be needed in order to see whether increased fear responses are a precursor to PTSD symptoms. Finally, there are multiple types of developmental change unfolding across this age range, and we are not able to assess all mechanisms that may contribute to changes in fear learning. By using well-defined paradigms like FPS with different groups, however, it is possible to elucidate how specific factors (e.g., violence exposure) interact with normative developmental change (e.g., pubertal development) to impact fear learning.

This study suggests that there are gene $\times$ environment $\times$ development effects of $A D C Y A P 1 R 1$ on conditioned fear responses in a high-risk pediatric population. Trauma interacted with genotype, in that those with the CC genotype and high levels of violence exposure showed amplified FPS in adolescence. Additional research, including pharmacological manipulations and neuroimaging, will be needed to elucidate the mechanisms that underpin these interactions during adolescence. Future studies with larger sample sizes, aimed at understanding how these interactions relate to intermediate phenotypes underlying traumarelated disorders, may be important for further understanding the development of PTSD and related syndromes.

\section{FUNDING AND DISCLOSURE}

This work was supported by funding from the NIH to TJ R01MH100122 and R01MH111682, and Brain and Behavior Research Foundation. KR has support from NIH R01MH112956, R01MH106595, R01HD088931, R01MH096764 and. NN effort is supported by $\mathrm{R} 01 \mathrm{MH} 108641$ and R01105379. The authors declare no competing interests.

\section{ACKNOWLEDGEMENTS}

The authors are grateful to the staff of the Grady Trauma Project for assistance in data collection, and the families that participated in the research.

\section{AUTHOR CONTRIBUTIONS}

TJ contributed to experimental design, data analysis, data interpretation writing first draft of manuscript, and finalizing the manuscript for submission; AFS contributed to data analyses and writing of manuscript; NT, AC, AC, and SM contributed to data collection, data analysis, and reviewed the manuscript; SJF and JSS contributed to design, data analysis and interpretation, and writing of the paper; AL contributed to all genetic data analyses and interpretation of data, as well as manuscript writing; NN contributed to statistical design and data interpretation and manuscript review; CFG contributed to data interpretation and manuscript preparation; BB contributed to experimental design and data interpretation; KJR contributed to experimental design, interpretation of results and reviewing and editing of the final version of the manuscript. 


\section{REFERENCES}

1. Norrholm SD, Glover EM, Stevens JS, Fani N, Galatzer-Levy IR, Bradley B, et al. Fear load: The psychophysiological over-expression of fear as an intermediate phenotype associated with trauma reactions. Int J Psychophysiol. 2015; 98:270-5.

2. Orr SP, Metzger LJ, Lasko NB, Macklin ML, Peri T, Pitman RK. De novo conditioning in trauma-exposed individuals with and without posttraumatic stress disorder. J Abnorm Psychol. 2000;109:290-8.

3. Peri T, Ben-Shakhar G, Orr SP, Shalev AY. Psychophysiologic assessment of aversive conditioning in posttraumatic stress disorder. Biol Psychiatry. 2000;47:512-9.

4. Duits P, Cath DC, Lissek S, Hox JJ, Hamm AO, Engelhard IM, et al. Updated metaanalysis of classical fear conditionoing in the anxiety disorders. Depression Anxiety. 2015;32:239-53.

5. Jovanovic T, Nylocks KM, Gamwell KL, Smith A, Davis TA, Norrholm SD, et al. Development of fear acquisition and extinction in children: Effects of age and anxiety. Neurobiol Learn Mem. 2014;113:135-42.

6. Jovanovic T, Nylocks KM, Gamwell KL, et al. Development of fear acquisition and extinction in children: effects of age and anxiety. Neurobiol Learn Mem. 2014;113:135-42.

7. Glenn CR, Klein DN, Lissek S, Britton JC, Pine DS, Hajcak G. The development of fear learning and generalization in 8-13 year-olds. Developmental Psychobiol. 2012;54:675-84.

8. van Rooij SJH, Cross D, Stevens JS, Vance LA, Kim YJ, Bradley B, et al. Maternal buffering of fear-potentiated startle in children and adolescents with trauma exposure. Soc Neurosci. 2017;12:22-31.

9. Pattwell SS, Duhoux S, Hartley CA, Johnson DC, Jing D, Elliott MD, et al. Altered fear learning across development in both mouse and human. Proc Natl Acad Sci. 2012;109:16318-23.

10. Schiele MA, Reinhard J, Reif A, Domschke K, Romanos M, Deckert J, et al. Developmental aspects of fear: Comparing the acquisition and generalization of conditioned fear in children and adults. Developmental Psychobiol. 2016;58:471-81.

11. Waters AM, Theresiana C, Neumann DL, Craske MG. Developmental differences in aversive conditioning, extinction, and reinstatement: a study with children, adolescents, and adults. J Exp Child Psychol. 2017;159:263-78.

12. Lau JY, Britton JC, Nelson EE, Angold A, Ernst M, Goldwin M, et al. Distinct neural signatures of threat learning in adolescents and adults. Proc Natl Acad Sci USA. 2011;108:4500-5.

13. Craske MG, Wolitzky-Taylor KB, Mineka S, Zinbarg R, Waters AM, VrshekSchallhorn $\mathrm{S}$, et al. Elevated responding to safe conditions as a specific risk factor for anxiety versus depressive disorders: Evidence from a longitudinal investigation. J Abnormal Psychol. 2012;121:315-24.

14. Gao Y, Raine A, Venables PH, Dawson ME, Mednick SA. The development of skin conductance fear conditioning in children from ages 3 to 8 years. Developmental Sci. 2010;13:201-12.

15. Paus T, Keshavan M, Giedd JN. Why do many psychiatric disorders emerge during adolescence? Nat Rev Neurosci. 2008;9:947-57.

16. Cross D, Vance LA, Kim YJ, Ruchard AL, Fox NA, Jovanovic T, et al. Trauma exposure, ptsd, and parenting in a community sample of low-income, predominantly african american mothers and children. Psychol Trauma. 2017;10:327-35.

17. van Rooij SJH, Jovanovic T. Impaired inhibition as an intermediate phenotype for PTSD risk and treatment response. Prog Neuro-Psychopharmacol Biol Psychiatry. 2019;89:435-45.

18. Lau JF, Pine D. Elucidating risk mechanisms of gene-environment interactions on pediatric anxiety: integrating findings from neuroscience. Eur Arch Psychiatry Clin Neurosci. 2008;258:97-106.

19. Sharma S, Ressler KJ. Genomic updates in understanding PTSD. Prog NeuroPsychopharmacol Biol Psychiatry. 2019;90:197-203.

20. Duncan LE, Cooper BN, Shen H. Robust findings from 25 years of PSTD genetics research. Curr Psychiatry Rep. 2018;20:115.

21. Lowe SR, Pothen J, Quinn JW, Rundle A, Bradley B, Galea S, et al. Gene-by-socialenvironment interaction ( $g x s e$ ) between adcyap1r1 genotype and neighborhood crime predicts major depression symptoms in trauma-exposed women. J Affect Disord. 2015;187:147-50.

22. Almli LM, Mercer KB, Kerley K, Feng H, Bradley B, Conneely KN, et al. Adcyap1r1 genotype associates with post-traumatic stress symptoms in highly traumatized african-american females. Am J Med Genet Part B, Neuropsychiatr Genet. 2013;162B:262-272.

23. Uddin M, Chang S-C, Zhang C, Ressler K, Mercer KB, Galea S, et al. Adcyap1r1 genotype, posttraumatic stress disorder, and depression among women exposed to childhood maltreatment. Depression Anxiety. 2013;30:251-8.
24. Wang L, Cao C, Wang R, Qing Y, Zhang J, Zhang XY. PAC1 receptor (ADCYAP1R1) genotype is associated with PTSD's emotional numbing symptoms in Chinese earthquake survivors. J Affect Disord. 2013;150:156-9.

25. Pohlack ST, Nees F, Ruttorf M, Cacciaglia R, Winkelmann T, Schad LR, et al. Neural mechanism of a sex-specific risk variant for posttraumatic stress disorder in the type i receptor of the pituitary adenylate cyclase activating polypeptide. Biol Psychiatry. 2015;78:840-847.

26. Stevens JS, Almli LM, Fani N, Gutman DA, Bradley B, Norrholm SD, et al. Pacap receptor gene polymorphism impacts fear responses in the amygdala and hippocampus. Proc Natl Acad Sci USA. 2014;111:3158-463.

27. Ressler KJ, Mercer KB, Bradley B, Jovanovic T, Mahan A, Kerley K, et al. Posttraumatic stress disorder is associated with pacap and the pac1 receptor. Nature. 2011;470:492-7.

28. Kirry AJ, Herbst MR, Poirier SE, Maskeri MM, Rothwell AC, Twining RC, et al. Pituitary adenylate cyclase-activating polypeptide (pacap) signaling in the prefrontal cortex modulates cued fear learning, but not spatial working memory, in female rats. Neuropharmacology. 2018;133:145-54.

29. Lind MJ, Marraccini ME, Sheerin CM, Bountress K, Bacanu S-A, Amstadter AB, et al. Association of posttraumatic stress disorder with $r s 2267735$ in the adcyap1r1 gene: A meta-analysis. J Trauma Stress. 2017;30:389-98.

30. Jovanovic T, Norrholm SD, Davis J, Mercer KB, Almli L, Nelson A, et al. Pac1 receptor (adcyap1r1) genotype is associated with dark-enhanced startle in children. Mol Psychiatry. 2012;18:742-3.

31. Chen W, Boutaoui N, Brehm JM, Han Y-Y, Schmitz C, Cressley A, et al. Adcyap1r1 and asthma in puerto rican children. Am J Respiratory Crit Care Med. 2013;187:584-8.

32. Gamwell KL, Nylocks M, Cross D, Bradley B, Norrholm SD, Jovanovic T. Fear conditioned responses and ptsd symptoms in children: Sex differences in fearrelated symptoms. Dev Psychobiol. 2015;57:799-808.

33. Purcell S, Neale B, Todd-Brown K, Thomas L, Ferreira MAR, Bender D, et al. Plink: A tool set for whole-genome association and population-based linkage analyses. Am J Hum Genet. 2007;81:559-75.

34. Fox NA, Leavitt LA. The Violence Exposure Scale for Children - VEX (preschool version). College Park, MD: Department of Human Development, University of Maryland; 1995.

35. Steinberg A, Brymer M, Decker K, Pynoos R. The University of California at Los Angeles post-traumatic stress disorder reaction index. Curr Psychiatry Rep. 2004;6:96-100.

36. Robertson EB, Skinner ML, Love MM, Elder GH, Conger RD, Dubas JS, et al. The pubertal development scale. J Early Adolescence. 1992;12:174-86.

37. Jackson F, Nelson BD, Meyer A, Hajcak G. Pubertal development and anxiety risk independently relate to startle habituation during fear conditioning in 8-14 yearold females. Developmental Psychobiol 2017;59:436-448.

38. Zuj DV, Norrholm SD. The clinical applications and practical relevance of human conditioning paradigms for posttraumatic stress disorder. Prog NeuroPsychopharmacol Biol Psychiatry. 2018;88:339-351.

39. Glenn DE, Acheson DT, Geyer MA, Nievergelt CM, Baker DG, Risbrough VB, et al. Fear learning alterations after traumatic brain injury and their role in development of posttraumatic stress symptoms. Depression Anxiety. 2017:34:723-33.

40. Zahn-Waxler C, Shirtcliff EA, Marceau K. Disorders of childhood and adolescence: gender and psychopathology. Annu Rev Clin Psychol. 2008;4:275-303.

41. Herringa RJ. Trauma, PTSD, and the developing brain. Curr Psychiatry Rep. 2017;19:69.

42. Wolf RC, Herringa RJ. Prefrontal-amygdala dysregulation to threat in pediatric posttraumatic stress disorder. Neuropsychopharmacology. 2016;41:822-831.

43. Gee DG, Humphreys KL, Flannery J, Goff B, Telzer EH, Shapiro M, et al. A developmental shift from positive to negative connectivity in human amygdala-prefrontal circuitry. J Neurosci. 2013;33:4584-93.

44. Mercer KB, Dias B, Shafer D, Maddox SA, Mulle JG, Hu P, et al. Functional evaluation of a ptsd-associated genetic variant: Estradiol regulation and adcyap1r1. Transl Psychiatry. 2016;6:e978.

45. McLaughlin KA, Sheridan MA, Gold AL, Duys A, Lambert HK, Peverill M, et al. Maltreatment exposure, brain structure, and fear conditioning in children and adolescents. Neuropsychopharmacology : official publication of the American College of Neuropsychopharmacology. 2016;41:1956-64.

46. Wolitzky-Taylor K, Vrshek-Schallhorn S, Waters AM, Mineka S, Zinbarg RE, Ornitz $\mathrm{EM}$, et al. Adversity in early and midadolescence is associated with elevated startle responses to safety cues in late adolescence. Clin Psychological Sci. 2014:2:202-13. 